\title{
Extirpación laparoscópica de quiste paraovárico gigante en pediatría
}

\author{
DOI 10.5377/alerta.v5i1.12802 \\ José Roberto González Ramos ${ }^{1 *}$, Jorge Márquez ${ }^{2}$ \\ 1 y 2. Hospital Nacional San Rafael, Santa Tecla, La Libertad, El Salvador \\ ${ }^{*}$ Correspondencia \\ $\square$ drgonzalezramos@gmail.com \\ (1) 0000-0001-9228-5653
}

\section{ACCESO ABIERTO}

Laparoscopic resection of a giant paraovarian cyst in pediatrics

\section{Citación recomendada:}

González Ramos JR, Márquez

JA. Extirpación laparoscópica de quiste paraovárico gigante en pediatría. Alerta. 2022:5(1):6-11. DO|: $10.5377 /$ alerta.v5i1.12802

\section{Recibido:}

4 de octubre de 2021.

\section{Aceptado:}

14 de diciembre de 2021.

\section{Publicado:}

27 de enero de 2022

\section{Contribución de autoría:} JRGR': revisión bibliográfica y elaboración de la discusión.

JAM$^{2}$ : elaboración del resumen clínico y revisión bibliográfica.

\section{Conflicto de intereses:}

Los autores declaran no tener ningún tipo de conflicto de interés.

\begin{abstract}
Resumen
Presentación del caso. Paciente femenina de 11 años con historia de un año de crecimiento abdominal progresivo, acompañado de dolor tipo cólico. En la evaluación física se encontró una masa en el hemiabdomen derecho, de consistencia blanda y dolorosa a la palpación profunda. Los estudios ultrasonográficos y la tomografía axial computarizada abdominopélvica confirmaron el diagnóstico de una masa quística de origen ovárico derecho con medidas de $13 \times 9 \mathrm{~cm}$. Intervención terapéutica. En la exploración laparoscópica se detectó el origen paraovárico del quiste y se procedió a la resección del quiste y se decidió la salpingectomía ipsilateral con preservación del ovario derecho. Evolución clínica. Se inició la alimentación por vía oral seis horas posteriores a la intervención. Se manejó con analgesia y fue dada de alta al día siguiente. Se continuó con el seguimiento ambulatorio por uno y tres meses posteriores a la cirugía sin detectarse complicaciones. La biopsia reportó un cistoadenoma seroso papilar paraovárico derecho y la ultrasonografía de control no mostró recidivas.

Palabras clave

Laparoscopía, pediatría, quiste paraovárico
\end{abstract}

\section{Abstract}

Case presentation. An 11-year-old female patient with a history of one year. In the clinical evaluation a mass was located in the right hemiabdomen, it had soft consistency and painful to deep palpation. Ultrasonographic and abdominopelvic computed tomography studies confirmed the diagnosis of a cystic mass of right ovarian origin. The mass measured $13 \times 9 \mathrm{~cm}$. Treatment. A video-laparoscopy defined the paraovarian cyst and proceeded its resection and ipsilateral salpingectomy and preservation of the right ovary. Outcome. Post-operative nutrition started after six hours. The patient had analgesic treatment and was discharged the following day. Follow-up was performed in an outpatient clinic at one month and at three months later, without complications. In the last control ultrasound was performed, which did not show any recurrence.

Keywords

Laparoscopy, paraovarian cyst, pediatrics

\section{Introducción}

El quiste paraovárico se desarrolla en el ligamento ancho, entre la trompa de Falopio y el ovario'. Son estructuras redondeadas de pared lisa, llenas de líquido seroso, que se ubican en el mesosálpinx, el ligamento ancho o sobre la propia trompa uterina. Tienen una incidencia aproximada de 1 en

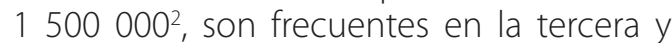
cuarta década de la vida; raramente se presentan en la edad pediátrica. Generalmente, son benignos ${ }^{1}$ y tiene un crecimiento lento y progresivo, suelen ser asintomáticos por lo que diagnóstico suele ser incidental ${ }^{3}$. En casos de quistes de gran volumen, los síntomas son dolor pélvico o la tumoración abdominal ${ }^{4}$. Usualmente son diagnosticados clínicamente y se confirman por imágenes ultrasonográficas ${ }^{5}$. La importancia de este caso es la poca frecuencia de estos tumores en la edad pediátrica y su identificación oportuna por parte de los profesionales de la salud. 


\section{Presentación del caso}

Paciente femenina de 11 años, referida por médico particular, con historia de aproximadamente un año de notar crecimiento progresivo de hemiabdomen derecho, que se acompañaba de dolor abdominal de tipo cólico de leve a moderada intensidad que cedía espontáneamente. Negó otros síntomas como fiebre, vómitos, estreñimiento, sintomatología urinaria, trastornos menstruales y pérdida de peso. Con antecedentes de menarquía a los diez años y su última menstruación un mes previo a la consulta. No hubo antecedentes familiares de importancia.

En la evaluación clínica, se encontró una paciente hemodinámicamente estable con frecuencia cardíaca de 84 latidos por minuto, frecuencia respiratoria de 20 respiraciones por minuto y tensión arterial de 100/60 mmHg. El abdomen se observó globoso, sin cicatrices ni circulación complementaria, se encontraba blando y depresible con peristaltismo presente y normal y se palpó una masa circunscrita, poco móvil, de aproximadamente $12 \times 12 \mathrm{~cm}$ que com- prometía el flanco derecho, mesogastrio, hipogastrio y fosa iliaca derecha, dolorosa a la palpación profunda y de consistencia blanda. No se observaron signos de irritación peritoneal. Los genitales externos femeninos sin anormalidades ni restos hemáticos. Presentó un estadio de Tanner III de desarrollo puberal, en el resto del examen físico no se encontraron anormalidades.

Los exámenes de laboratorio reportaron: hemoglobina de 13,5 gr/dL, hematocrito de $40 \%$, glóbulos blancos de $7800 \times \mathrm{mm}^{3}$, neutrófilos de $65 \%$, linfocitos de $30 \%$, tiempo parcial de tromboplastina de 32 segundos, tiempo y valor de protrombina de 12 segundos, $90 \%$, examen general de orina sin anormalidades, antígeno carcinógeno 125 de 16,6 U/ml, antígeno carcinoembrionario de 2,6 ng/ml, alfafetoproteína de 0,54 Ul/ml y hormona gonadotropina coriónica fracción beta de 0,98 mUl/ml.

La ultrasonografía pélvica y la tomografía axial computarizada abdominopélvica describieron una masa quística $13 \times 9 \mathrm{~cm}$, de contenido líquido, con un septo intracavitario sin componentes sólidos. El ovario izquierdo con características normales y el ovario derecho no se observó (Figura 1).

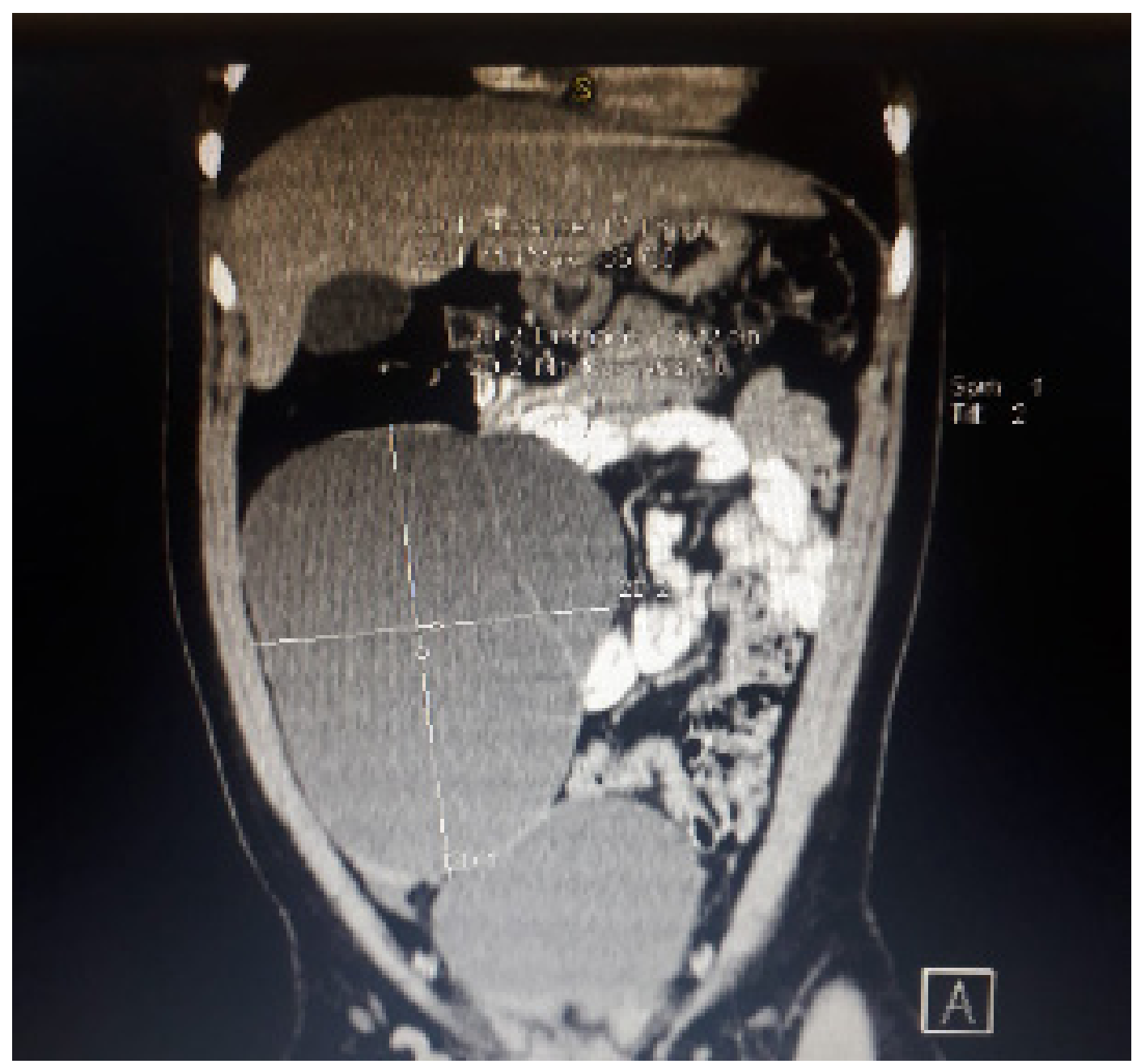

Figura 1. Tomografía axial que muestra una masa quística del anexo derecho con septo en su interior, que desplaza las asas intestinales. 


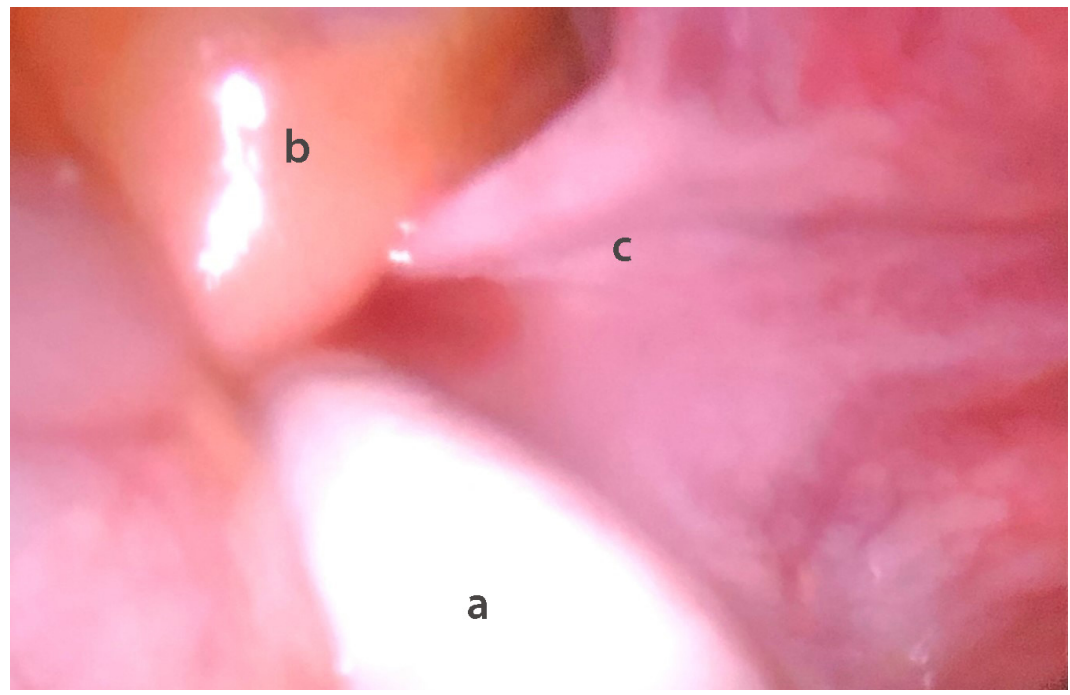

Figura 2. Se observa ovario derecho (a), el útero (b) y la trompa de Falopio (c).

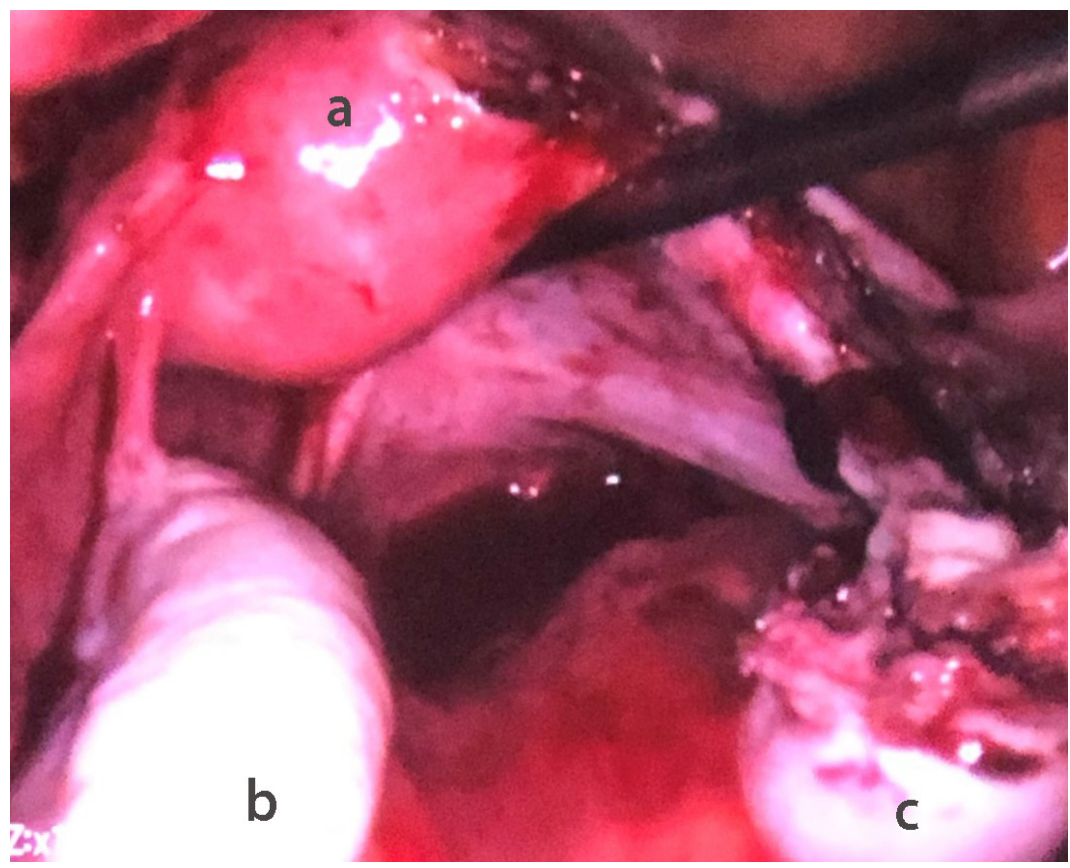

Figura 3. Se observa el útero (a) y ambos ovarios (b y c) al final de la cirugía.

Al tener los estudios, se realizó la intervención quirúrgica dos semanas después. Durante este tiempo no recibió ningún tratamiento médico.

\section{Intervención terapéutica}

La paciente ingresó al servicio de pediatría un día antes de su intervención quirúrgica. Se realizó profilaxis con cefazolina 1 gr por vía intravenosa, 30 minutos antes del inicio de la cirugía.

El procedimiento quirúrgico fue una extirpación laparoscópica. Inicialmente se intentó establecer neumoperitoneo por técnica abierta a través de incisión umbilical en la que se colocó un trocar de $10 \mathrm{~mm}$ en el ombligo, pero el tamaño de la masa no lo permitió, por lo que se puncionó el quis- te con una aguja de Veress y se drenaron $1200 \mathrm{ml}$ de líquido claro, citrino, sin restos macroscópicos. Esto permitió formar el neumoperitoneo con presión abdominal de 12 $\mathrm{mmHg}$ y flujo inicial de $5 \mathrm{l} / \mathrm{min}$. Se colocó un trócar de $5 \mathrm{~mm}$ el flanco inferior derecho y otro en el flanco inferior izquierdo, luego se identificó la masa proveniente del mesosálpinx derecho, que comprometía la trompa uterina ipsilateral y se observaron ambos ovarios sanos (Figura 2 y 3 ).

\section{Evolución clínica}

La paciente recibió tratamiento con ketorolaco $30 \mathrm{mg}$ y paracetamol $500 \mathrm{mg}$ cada seis horas, ambas por vía intravenosa e inició la alimentación por vía oral seis horas posteriores a la cirugía. Se dio el alta hospitalaria al día siguiente con ibuprofeno $400 \mathrm{mg}$ cada ocho horas y sin antibióticos. Se dio seguimiento una semana, un mes y tres meses posteriores a la cirugía. En el control semanal se reportó la biopsia que mostró un cistoadenoma seroso papilar paraovárico derecho. En el último control se tomó una ultrasonografía pélvica sin encontrarse evidencia de recidiva del quiste.

\section{Diagnóstico clínico}

Cistoadenoma seroso papilar paraovárico derecho.

\section{Discusión}

Los quistes paraováricos representan aproximadamente el $10 \%$ de las lesiones anexiales diagnosticadas y el $3 \%$ de todas las urgencias ginecológicas. Son de origen mesotelial en el $68 \%$ de los casos y se presentan raramente en la edad pediátrica ${ }^{5,7}$. Se clasifican histológicamente como quistes serosos o simples, su tamaño habitual es de uno a ocho $\mathrm{cm}$, pero se han descrito quistes mayores de diez $\mathrm{cm}$ de diámetro ${ }^{6}$. Esta descripción de las características anatomohistológicas de los quistes paraováricos es coincidente con los hallazgos de nuestra paciente.

Se desconoce la etiología de los quistes paraováricos, es probable que se deba a la acción secretora del epitelio tubárico, como resultado de la actividad hormonal después de la pubertad ${ }^{8}$. Hay pocos informes de quistes paraováricos en pacientes con insuficiencia ovárica prematura, que se caracteriza por bajos niveles de estrógeno y andrógenos ${ }^{8}$.

Estos suelen ser unilaterales, móviles y lisos. La sintomatología clínica de estas pa- 
cientes se puede manifestar de manera crónica o aguda. La primera se caracteriza por presentar frecuentemente síntomas digestivos, como la sensación de pesantez en la parte inferior del abdomen junto con dolor, que puede ser ligero, pasajero o en ocasiones frecuente, pero tolerable. Otras jóvenes manifiestan sintomatología urinaria como la polaquiuria, acompañada algunas veces por fiebre ${ }^{5,9}$. Por otra parte, el cuadro agudo se puede caracterizar con sensibilidad abdominal marcada producida por hemorragias intraquísticas, perforación del quiste con hemoperitoneo o la torsión de la tumoración o de la trompa de Falopio del mismo lado. El diagnóstico diferencial del quiste paraovárico debe incluir: la duplicación intestinal, las hernias internas, el linfangioma intestinal y los quistes de inclusión peritoneal. Ante un cuadro abdominal agudo debe descartarse la apendicitis complicada de varios días de evolución, el hidrosálpinx, la torsión ovárica y el embarazo ectópico $5,6,9$.

La recurrencia, parece suceder más comúnmente en el mismo lado. Parece no asociarse a otras patologías, así como tampoco el tamaño del quiste, su número, el índice de masa corporal o la etapa de pubertad ${ }^{8}$.

Es difícil establecer el diagnóstico preoperatorio preciso de quistes paraováricos. Inicialmente el cuadro clínico es inespecífico y cuando se complica se manifiesta como un cuadro abdominal agudo. Radiológicamente resulta complejo distinguirlos de los cistoadenomas de ovario, quistes de ovario o de los quistes foliculares. La primera aproximación diagnóstica es la ecografía. Se debe sospechar la presencia de quiste paraovárico cuando se observa un quiste localizado en el lado del útero y no se consigue identificar ambos ovarios ${ }^{6,12}$. La tomografía computarizada y la resonancia magnética presentan limitaciones en determinar el origen de los quistes paraováricos de gran tamaño. Solo de un 30 a un $44 \%$ de los quistes paraováricos son identificados correctamente antes de la cirugía $6,9,10,12$.

En el estudio previo a la intervención quirúrgica es importante la determinación de marcadores tumorales para diferenciar entre tumores malignos de benignos ${ }^{13}$. Los marcadores tumorales de mayor utilidad para descartar malignidad incluyen alfafetoproteína, beta gonadotropina coriónica humana, antígeno de cáncer 125 y lactato deshidrogenasa. Los marcadores tumorales de bajo rendimiento incluyen la inhibina $A$ y $B^{14}$.

Las complicaciones del quiste paraovárico, como la hemorragia, la perforación, la torsión y el riesgo de malignización, se deben al rápido crecimiento y gran tamaño y se manifiestan como cuadros abdominales agudos. La malignización ocurre en el 2,9\% de los casos y se describe el cistoadenocarcinoma y el carcinoma papilar como las principales neoplasias secunadarias 6 . A largo plazo hay un aumento del riesgo de embarazos ectópicos ${ }^{10}$. La torsión anexial es una complicación común que se diagnostica en niñas sometidas a cirugía por quistes paraováricos y no se asocia con la apariencia ecográfica o el diámetro del quiste. Por lo tanto, para prevenir la torsión, se debe considerar la extirpación quirúrgica de los quistes paraováricos en las niñas que se someten a otras intervenciones y se encuentran como hallazgos fortuitos durante esta ${ }^{14}$. Estas intervenciones quirúrgicas deben ser cautelosas, procurando conservar tanto la trompa de Falopio, así como el ovario involucrado en función de preservar la fertilidad y la función hormonal ${ }^{17}$. En este caso no fue posible conservar la trompa debido a que, por el tamaño del quiste, no se pudo establecer un plano quirúrgico seguro para su disección, pero se logró preservar el ovario derecho como se muestra en la Figura 3. Otro punto importante es la conducta quirúrgica frente al hallazgo de torsión y necrosis de los tejidos anexiales. Normalmente al observarse macroscópicamente la evidencia de necrosis del ovario y anexos, se toma la decisión de la extirpación durante la cirugía, pero existen reportes que muestran un manejo expectante en estos casos con recuperación funcional completa después de desenrollar los anexos isquémicos independientemente de la apariencia macroscópica ${ }^{12,13}$. Es por ello que se recomienda la extirpación del quiste en el hallazgo incidental durante una cirugía realizada por otros motivos ${ }^{10}$. La naturaleza benigna de la masa se confirma por estudio histológico 6,10,11.

El tratamiento quirúrgico de la masa anexiales puede realizarse a través de una laparotomía o una laparoscopía. Esto depende de factores asociados tanto al paciente como al tumor. Las ventajas de la laparoscopia son las siguientes: la menor molestia postoperatoria, pérdidas sanguíneas menores, menor trauma de los tejidos, disminución de la estancia hospitalaria y menores costos de atención ${ }^{12}$. Sus desventajas se presentan en casos de patología maligna; si el tamaño del quiste es muy grande o si se carecen de las habilidades o equipo necesario para realizar esta vía de forma completa ${ }^{12,17}$.

Están descritos varios métodos de abordaje de los quistes gigantes, tales como el drenaje transdérmico bajo control ultrasonográfico; la descompresión del quiste por minilaparotomía y la aspiración del quiste bajo control laparoscópico ${ }^{17}$, tal como se hizo en este caso. 
Existen alternativas a la cirugía laparoscópica tradicional como la cirugía de puerto único LESS (Laparoendoscopy Single Site) y la cirugía laparoscópica isobárica. La cirugía de puerto único consiste en realizar una incisión umbilical única a través de la cual, por medio de un trócar diseñado para tal fin, se introduce el instrumental necesario para realizar el procedimiento ${ }^{16}$.

Por otro lado, la cirugía laparoscópica isobárica consiste en crear el espacio de trabajo abdominal con retractores de la pared abdominal y no con dióxido de carbono $17,18,19$.

La progresión tecnológica de la ciencia médica ha permitido la utilización de tecnología robótica para la extirpación de masas anexiales en la edad pediátrica y ha evidenciado ser un procedimiento quirúrgico simple, seguro y eficaz para pacientes seleccionadas $^{20}$.

\section{Aspectos éticos}

Para la publicación de este caso se obtuvo el consentimiento informado de los responsables de la paciente tanto para la atención hospitalaria en la que se realizó el examen físico de la paciente, así como para la publicación de este artículo, con el compromiso de mantener la privacidad de la paciente, según lo establecido en la declaración de Helsinki.

\section{Agradecimiento}

Al Dr. Sidney Abarca, médico ginecólogo del Hospital Nacional San Rafael, por su apoyo en el procedimiento quirúrgico.

\section{Financiamiento}

Recursos propios de los autores.

\section{Referencias bibliográficas}

1. Durairaj A, Gandhiraman K. Complications and management of paraovarian cyst: a retrospective analysis. J Obstet Gynaecol India. 2019;69(2):180-184. DOI: $10.1007 /$ s13224-018-1152-2

2. Katke RD, Gohil D. Rupture and torsion of a huge ovarian cyst with ipsilateral para-ovarian cyst presented as haemoperitoneum and acute abdomen: a rare case report and review of literature. Int J Res Health Sci. 2014;31;2(4):11703. Disponible en: https://ijrhs.org/ article/2014/2/4-26
3. De Sanctis V, Soliman AT, Elsedfy H, Soliman $N$, Elalaily R, Di Maio S, et al. An adolescent with an asymptomatic adnexal cyst: to worry or not to worry? Medical versus surgical management options. Acta Biomed. 2017;88(2):232-236. DOI: 10.23750/abm. v88i2.6050

4. Kiseli M, Caglar GS, Cengiz SD, Karadag

D, YIlmaz MB. Clinical diagnosis and complications of paratubal cysts: review of the literature and report of uncommon presentations. Arch Gynecol Obstet. 12;285(6):15639. DOI: $10.1007 / 500404-012-$ $\underline{2304-8}$

5. Jiménez JH, Rascón Alcantar A, Fimbres Franco R. Adolescente con quiste paraovárico. Tratamiento quirúrgico. Cir. 2017;85(6):535-538 DOI: 10.1016/j. circir.2016.08.002

6. Torres PJ, Îñiguez DR. Quiste paraovárico gigante en la infancia. Reporte de un caso. Rev Chil Pediatr. 2015;86(2):117-20. DOI: $\underline{10.1016 / j \text {.rchipe.2015.04.023 }}$

7. Mărginean CO, Mărginean C, Meliț LE, Săsăran Vş, Poruțiu M, Mărginean CD. An incidental diagnosis of a giant paraovarian cyst in a female teenager: A case report. Medicine (Baltimore). 2018;97(48): e13406. DOI: $10.1097 / M D .0000000000013406$.

8. Zhao Z, Zhou T, Zhao S, Zhang W, Rong F. Case report of a paraovarian cyst in a 15-year-old adolescent girl with premature ovarian failure. Int J Gynaecol Obstet. 2017;139(2):245-6. DOI: $10.1002 /$ ijgo.12265

9. Zvizdic Z, Bukvic M, Murtezic S, Skenderi

F, Vranic S. Giant paratubal serous cystadenoma in an adolescent female: Case report and literature review. J Pediatr Adolesc Gynecol. 2020;33(4):438-40. DOI: 10.1016/j.jpag.2020.03.010

10. Magistrado L, Dorland J, Sangi-Haghpeykar $\mathrm{H}$, Patil N, Dietrich JE. Paratubal cyst recurrence in children and adolescents. J Pediatr Adolesc Gynecol. 2020;33(6):649-51. DOI: 10.1016/j.jpag.2020.07.008

11. Fujishita A, Araki H, Yoshida S, Hamaguchi D, Nakayama D, Tsuda N, et al. Outcome of conservative laparoscopic surgery for adnexal torsion through one-stage or two-stage operation: Laparoscopic surgery for adnexal torsion. J Obstet Gynaecol Res. 2015;41(3):411-7. DOI: 10.1111/jog.12534

12. Mărginean $C O$, Mărginean $C$, Chinceşan M, Mărginean MO, Meliț LE, Săsăran V, et al. Pediatric ovarian tumors, a challenge for pediatrician and gynecologist: Three case reports (CARE compliant). Medicine (Baltimore). 2019;98(16): e15242. DOI: 10.1097/MD.0000000000015242

13. Tzur T, Smorgick N, Sharon N, Pekar-Zlotin M, Maymon R, Melcer Y. Adnexal torsion with paraovarian cysts in pediatric and adolescent populations: A retrospective 
study. J Pediatr Surg. 2021;56(2):324-7. DOI: 10.1016/i.jpedsurg.2020.05.023

14. De Lambert G, Fresneau B, Chapelière S, Mussini C, Martelli H. Masas ováricas de la niña y la adolescente. EMC- Pediatría. 2018;53(2):1-12. DOI: 10.1016/s12451789(18)90865-6

15. Xac MC., Jetelina KK., Jarin J., Wilson E. Benign, borderline, and malignant pediatric adnexal masses: A 10-year review. J Pediatr Adolesc Gynecol. 2021;34(4):454-461. DOI: 10.1016/j.jpag.2021.01.002

16. Schmitt A, Crochet P, Knight S, Tourette C, Loundou A, Agostini A. Single-port laparoscopy vs conventional laparoscopy in benign adnexal diseases: A systematic review and meta-analysis. J Minim Invasive Gynecol. 2017;24(7):1083-1095. DOI: 10.1016/j.jmig.2017.07.001

17. Raźnikiewicz A, Korlacki W, Grabowski A. Evaluation of the usefulness of laparoscopy in the diagnosis and treatment of adnexal pathologies in the pediatric population. Wideochir Inne Tech Maloinwazyjne. 2020;15(2):366-376. DOI: 10.5114/ wiitm.2020.93998
18. Takeda A, Imoto S, Mori M, Nakano T, Nakamura H. Isobaric laparoendoscopic single-site surgery with wound retractor for adnexal tumors: a single center experience with the initial 100 cases. Eur J Obstet Gynecol Reprod Biol. 2011;157(2):190-6. DOI: 10.1016/j.ejogrb.2011.02.018

19. Levy L, Tsaltas J. Recent advances in benign gynecological laparoscopic surgery. Fac Rev. 2021; 10:60. DOI: 10.12703/r/10-60

20. Xie XX, Wang N, Wang ZH, Zhu YY, Wang $J R$, Wang $X Q$. Robotic-assisted resection of ovarian tumors in children: A case report and review of literature. World J Clin Cases. 2019; 717:2542-2548. DOI: 10.12998/wjcc. v7.117.2542 